\title{
Congruencies between photoautotrophic groups in springs of the Italian Alps: implications for conservation strategies
}

\author{
Juri NASCIMBENE*, Daniel SPITALE ${ }^{1)}$, Holger THÜS ${ }^{2)}$ and Marco CANTONATI ${ }^{1)}$ \\ University of Padova, Department of Biology, Via U. Bassi 58/B, 35121 Padova, Italy \\ ${ }^{1)}$ Museo Tridentino di Scienze Naturali (MTSN), Limnology and Phycology Section, Via Calepina 14, I-38122 Trento, Italy \\ ${ }^{2}$ Botany Department, The Natural History Museum, Cromwell Road, London SW7 5BD, United Kingdom \\ *e-mail corresponding author: junasc@libero.it
}

\begin{abstract}
Even though a number of studies have demonstrated the importance of photoautotrophic organisms in spring habitats, investigations that consider several photoautotrophic taxonomic groups are lacking. Within the framework of a multidisciplinary project on springs of the south-eastern Alps, we studied algae, diatoms, lichens, and bryophytes and (1) compared the alpha, beta and gamma diversity, and the composition of the studied groups between carbonate and siliceous springs, (2) estimated the nonrandomness of species combinations within organismal groups, and (3) examined the congruence in species assemblage patterns across taxonomic groups. In 40 springs, 69 species of algae, 110 species of diatoms, 29 species of lichens, and 62 species of bryophytes were found. Diatoms, lichens and bryophytes had higher species-richness in siliceous springs, while other algae had higher richness in carbonate springs. For all taxonomic groups, carbonate and siliceous springs host different assemblages, indicating that both types of substrata contribute to the overall regional diversity of spring photoautotrophs. In individual springs, the photoautotroph groups are characterised by a similar proportion of species of their regional pool, and form relatively speciespoor communities with a high turnover of species among springs. This pattern has important implications for conservation, suggesting that the protection of single sites might not be effective, and that a biodiversity conservation plan for spring habitats should be developed at the regional level, and include a network of sites. Interestingly, the co-occurrence indices suggested that, in individual springs, stochastic processes might the most important mechanisms in the establishment of local assemblages. A weak cross-taxon congruency was found, suggesting that a single taxon surrogate will not adequately represent other photoautotrophic groups. Therefore, spring conservation plans for photoautotrophs should not use one group as a surrogate for overall photoautotrophic diversity, but should adopt the use of different taxonomic groups.
\end{abstract}

Key words: springs, conservation, surrogates, multiple taxonomic groups, Autonomous Province of Trento

\section{INTRODUCTION}

The importance of springs for biodiversity is well recognized (Botosaneanu 1998; Cantonati et al. 2006). They often harbour highly diverse communities of animals and plants, and in some cases the biota shows a high degree of endemism (e.g., Hinterlang \& Lischewski 1996; Di Sabatino et al. 2003). Direct (e.g., water abstraction) and indirect (e.g., land use for cattle grazing) utilization of springs are beneficial for society, but these uses may also be associated with significant costs to the environment, including biodiversity loss (Barquin \& Scarsbrook 2008). Any effective management of springs will be only achieved by recognizing and preserving both the societal and the biological values of this habitat. Regrettably, while there is awareness of the economic value of high-quality water, the importance of biodiversity conservation has so far only been addressed by a few studies that comprehensively documented crenic biodiversity (e.g., Cantonati et al. 2006).

A number of studies have demonstrated the importance of photoautotrophic organisms in springs, e.g. diatoms (Sabater \& Roca 1990; Cantonati 1998), other algae (Cantonati 2008), lichens (Nascimbene et al.
2007) and bryophytes (Stream Bryophyte Group 1999). These studies have laid the foundations for more detailed ecological studies. For example, studies on crenic diatoms highlighted the role of these organisms in providing information on water quality (Aboal et al. 1998), hydraulic regime, discharge, shading and nitrates (Cantonati \& Spitale 2009), and for the identification of spring types (Cazaubon \& Rolland 1995). With respect to bryophytes, Spitale (2009) demonstrated that the extent of water-level fluctuations in springs could be inferred by analysing the spatial distribution of the species.

However, because of the difficulty of combining contrasting taxonomic expertise within a common framework, studies which consider all photoautotrophs are lacking in crenoecology. Based on our observations, we anticipated that a study of all photoautotrophs inhabiting springs would provide important insights into both the response of these organisms to the environment, and the potential interaction between different taxonomic groups. This information is crucial for developing a science-based strategy in spring conservation for photoautotrophs.

In the south-eastern Alps, springs were recently investigated in detail during a multidisciplinary project which aimed to evaluate their role in biodiversity con- 
servation, and to identify the main ecological factors influencing organisms. Within the framework of this project, we selected a pool of sites in which we studied four groups of photoautotrophs, algae (cyanoprokaryotes and algae other than diatoms), diatoms, lichens, and bryophytes. Our main aims in this study were (1) to compare the alpha, beta and gamma diversity, and the composition of the studied groups between carbonate and siliceous springs, (2) to estimate the non-randomness of species combinations among organismal groups, and (3) to examine the congruence in species assemblage patterns across taxonomic groups.

\section{METHODS}

\subsection{Study area}

The study was carried out in the Autonomous Province of Trento (NE Italy), and included 40 springs that are unimpacted by artificial structures for water abstraction, equably distributed across the study area. The altitudinal distribution ranges between 500 and $2700 \mathrm{~m}$ a.s.l., including the submediterranean (6 sites), montane ( 25 sites) and subalpine/alpine (9 sites) altitudinal belts. Twenty-one springs were on siliceous rocks and 19 on carbonate rocks. Following the geomorphological spring classification by Howein \& Schroeder (2006), which is based on substratum particlesize, they mainly belonged to cobble and boulder springs.

\subsection{Sampling}

Sampling was carried out in the whole spring area, including all wetted (micro-)habitats, with some methodological differences according to the ecology of each organism, as detailed below.

As used here, the term alga does not refer to a particular systematic entity, but comprises a variety of lower plants without true tissues but with macroscopically visible characters (colourings, covers, thalli) (e.g., Cantonati et al. 2007). They include Cyanophyta (cyanobacteria, cyanoprokaryota), Rhodophyta, Heterokontophyta (Chrysophyceae, Xanthophyceae), and Chlorophyta (Chlorophyceae, Zygnematophyceae). Algae were collected from all the available immersed or emergent microhabitats, from the spring mouth to no more than 5 metres downstream. When possible, fresh material was studied under a Zeiss Axioskop 2 microscope to document the individual taxa with digital images, and to measure diacritical characters. Detailed identifications and further digital images were obtained from material preserved in 2-3\% formaldehyde, using a Zeiss Axioskop 2 microscope equipped with a Zeiss Axiocam digital camera. The literature used for identification is listed in Cantonati (2008).

Diatoms were collected by brushing 10 quadrats $(3 \times 3 \mathrm{~cm})$ from at least 5 stones collected haphazardly from the submerged area, from the spring mouth to about 5 metres downstream. For each sample at least
500 valves were counted under a Zeiss Axioskop 2 at $1000 \times$ magnification. Some critical taxa were also examined with SEM (Zeiss-EVO40XVP) at the MTSN. Identification and nomenclatural details are reported elsewhere (Cantonati \& Spitale 2009).

Lichens are the symbiotic phenotypes of specialised heterotrophic fungi. The intact lichen thalli with their incorporated algae are functionally comparable to photoautotrophic organisms. Therefore lichens are treated here as photoautotrophic organisms. They were sampled from both perennially and periodically inundated stones within the first 5 metres of the spring mouth. Species nomenclature follows Nimis \& Martellos (2008), except for Verrucaria funckii (Thüs 2002).

Bryophytes were collected from immersed and emergent habitats from the spring mouth to about 5 metres downstream. The wetted, emergent habitats were within a few decimetres of the water's edge. Species were mainly identified according to Cortini-Pedrotti (2001, 2006) for mosses, and Paton (1999) for liverworts.

\subsection{Data analysis}

\subsubsection{Species-richness, diversity, and composition}

For each taxonomic group, differences in speciesrichness between carbonate and siliceous springs at the 40 sites were tested with a parametric $t$-test for unpaired samples. The alpha $(\alpha)$, beta $(\beta)$ and gamma $(\gamma)$ components of diversity (Allan 1975; Lande 1996; Koleff et al. 2003) were considered. In this work, $\gamma$ represents the total number of species found in each spring type (i.e., on carbonate and siliceous springs), $\alpha$ represents the mean number of species in individual springs, and $\beta$ was calculated using Sørensen's index, giving estimates of the turnover of the autotrophic biota within each type of spring.

We used species accumulation curves (Magurran 2004) to evaluate the cumulative number of species recorded in both carbonate and siliceous springs, as a function of the number of springs. Because the order in which samples are included influences the shape of the curve, samples are randomly added to the species accumulation curve repeating the procedure 99 times.

Using a matrix of presence/absence, differences in species composition between carbonate and siliceous springs for each taxonomic group were tested by a multi-response permutation procedure (MRPP), implemented in PC-ORD (McCune \& Grace, 2002; McCune $\&$ Mefford 1999) using the Sørensen distance measure and rank transformation of the distance matrices.

\subsubsection{Analysis of the group structure}

The co-occurrence patterns of taxonomic groups were evaluated using different indices, implemented in Ecosim 7.0 (Gotelli \& Entsminger 2001): (1) C-score, (2) V-ratio, (3) number of checkerboard pairs, and (4) number of species combinations (Combo). The C-score (1) measures the average number of "checkerboard 
units" between all possible pairs of species. Checkerboard means that, when for example bryophyte "A" is present, lichen "B" is absent. In a competitively structured community, the $\mathrm{C}$-score should be significantly larger than that expected by chance. The variance ratio (2) is considered as an index of variability in speciesrichness per site. If niche limitation constrains the number of coexisting species, the variance in species-richness among sites will be small relative to the null model. In a competitively structured community, the observed variance ratio should be significantly smaller than that expected by chance. The number of checkerboard pairs (3), is conceptually similar to the C-score, and tabulates the number of species pairs that never cooccur in any site. In a competitively structured community, there should be more checkerboard pairs of species than that expected by chance. In the number of species combinations, Combo (4), as the name suggests counts the number of unique species combinations between taxonomic groups represented at different sites. In a competitively structured community, there should be fewer species combinations than that expected by chance. The null models against which to compare the observed indices were generated by randomizing species presences of the observed matrices but maintaining the row and column sums (Gotelli 2000). Thus, in the simulated data, each spring will have exactly the same number of species as in the real data set. Similarly, each species will occur in the same number of springs as in the real data. The statistical significance was evaluated by comparing the value of the index $(1,2,3,4)$ obtained from the observed matrix to the index from the randomized matrices. The null hypothesis is rejected at a significance level of $p<0.05$.

\subsubsection{Congruency in species assemblage patterns across taxonomic groups}

Statistical analyses of similarity of assemblage composition, based on a presence/absence matrix, were performed using PRIMER version 6. Similarity was calculated between every pair of springs using the Sørensen similarity index (Legendre \& Legendre 1998). For every pair of similarity matrices, congruence was evaluated using Mantel tests, using the Spearman rank correlation method with 999 permutations. The Mantel analysis tests the null hypothesis of no relationship between multivariate patterns from two sets of samples, i.e. species assemblages in this study (Clarke \& Gorley 2001). The null hypothesis is rejected at a significance level of $p<0.05$. The Rho-value indicates the strength of the observed relationship with 0 denoting no relationship, 1 denoting perfect positive correlation between two triangular matrices.

\section{RESULTS}

\subsection{Species-richness and assemblages composition}

Sixty-nine species of algae, 110 species of diatoms, 29 species of freshwater lichens, and 62 species of bryophytes were found. For all photoautotrophic groups other than algae, a higher total number of species $(\gamma$ diversity) was found in siliceous springs (Tab. 1), the ratio of species-richness between the two substrata being highest for lichens (1.9) and lowest for bryophytes (1.3). A similar pattern was found in individual springs ( $\alpha$-diversity). Lichens were the most speciespoor group, followed by algae and mosses, while diatoms are the richest group. Species-richness of diatoms was significantly higher in siliceous springs, whereas other algae were significantly more species-rich in carbonate springs. Lichens and bryophytes tended to be more species-rich in siliceous springs, but the difference was not significant (Tab. 1). Also the heterogeneity of the autotrophic biota ( $\beta$-diversity) did not differ between the two types of springs, with the exception of algae, which had higher heterogeneity in siliceous springs.

Tab. 1. Species-richness and components of diversity for the four photoautotrophic groups in carbonate and siliceous springs of the Italian Alps. Unpaired $t$-test between carbonate (Car) and siliceous (Sil) springs; mean values are given with \pm Standard Deviation.

\begin{tabular}{lccc}
\hline Spring type & Car & Sil & $P$ \\
\hline$\gamma$-diversity & & & \\
Algae & 48 & 37 & - \\
Diatoms & 60 & 100 & - \\
Lichens & 11 & 21 & - \\
Bryophytes & 38 & 49 & - \\
$\alpha$-diversity & & & \\
Algae & $6.8 \pm 3.1$ & $3.6 \pm 2$ & 0.001 \\
Diatoms & $10.6 \pm 5$ & $15.5 \pm 7$ & 0.02 \\
Lichens & $1.5 \pm 0.8$ & $2.4 \pm 2.7$ & n.s. \\
Bryophytes & $6.4 \pm 3$ & $7.7 \pm 3$ & n.s. \\
$\beta$-diversity & & & \\
Algae & $58.7 \pm 1.8$ & $64.5 \pm 1.3$ & 0.017 \\
Diatoms & $46.3 \pm 2.4$ & $50.9 \pm 2.5$ & n.s. \\
Lichens & $39.7 \pm 8.5$ & $45.1 \pm 10.9$ & n.s. \\
Bryophytes & $50.2 \pm 2.4$ & $53.7 \pm 3.0$ & n.s. \\
\% species on the regional pool & & \\
Algae & $14.2 \pm 6.5$ & $9.9 \pm 5.4$ & 0.05 \\
Diatoms & $17.6 \pm 7.9$ & $15.5 \pm 7.3$ & n.s. \\
Lichens & $14.7 \pm 7.8$ & $12 \pm 13$ & n.s. \\
Bryophytes & $16.9 \pm 8.6$ & $14.9 \pm 7.6$ & n.s. \\
\hline
\end{tabular}

Diatoms, lichens, and bryophytes constitute a similar proportion of the regional species-pool ( $\gamma$-diversity) in siliceous and carbonate springs, but the proportion of algae was significantly smaller in siliceous springs.

Accumulation curves illustrate the pattern of diversity, showing that diatoms, and to lesser extent bryophytes (on siliceous substrata) and algae (on carbonate), were the most species-rich groups (Fig. 1). The rate at which new species are added to the total species-pool is higher for diatoms, algae and bryophytes than for lichens.

According to MRPP, for all photoautotrophic groups community composition differs significantly between carbonate and siliceous springs (Tab. 2). 

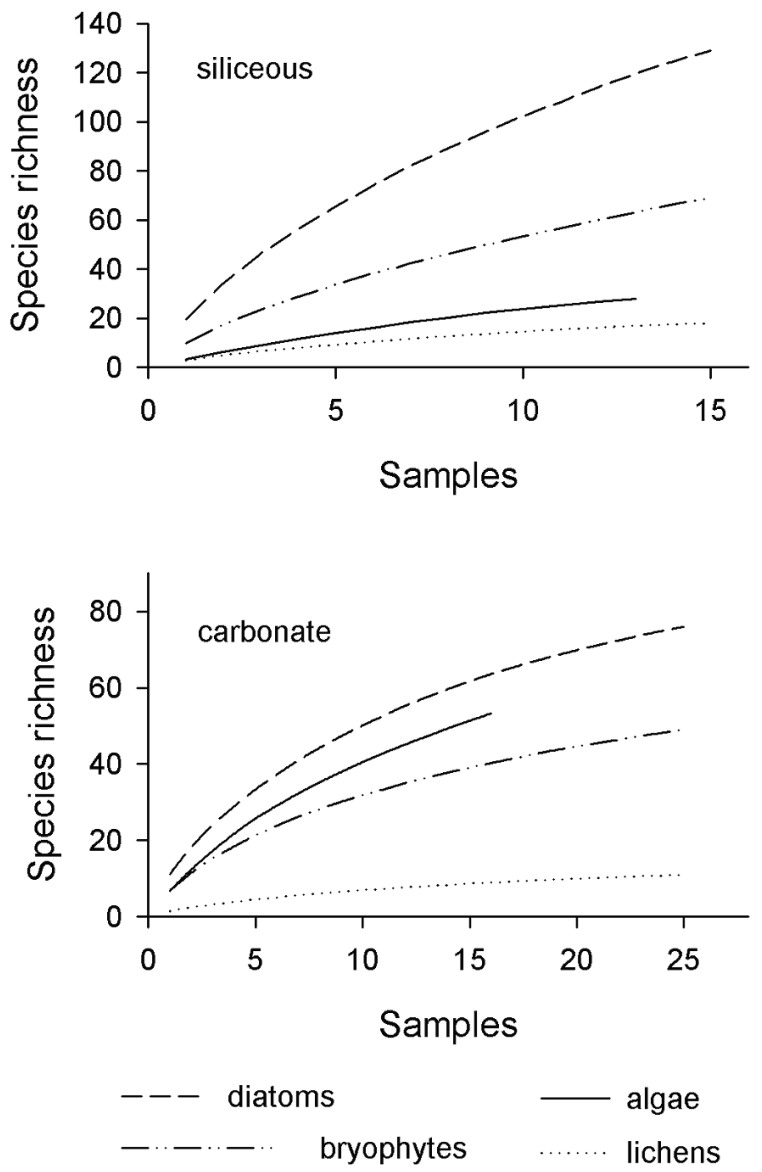

Fig. 1. Species accumulation curves of diatoms, algae, bryophytes, and lichens in siliceous and carbonate springs of the Italian Alps. Average species-richness is based on 99 randomized permutations.

Tab. 2. MRPP results. Effect size A-, and p-values for the non-metric multi-response permutation procedures (MRPP) applied to the two types of substrata (Carbonate vs Siliceous) for each group of photoautotrophs in springs of the Italian Alps. The test statistic "A" in MRPP describes the separation between groups (McCune \& Grace 2002). The higher the A value, the stronger the between-group difference $(p<0.05)$. In community ecology, values for A are commonly below 0.1 .

\begin{tabular}{lcc}
\hline Group of autotrophs & $\mathrm{A}$ & $\mathrm{p}$ \\
\hline Algae & 0.05 & 0.020 \\
Diatoms & 0.04 & 0.013 \\
Lichens & 0.32 & $<0.001$ \\
Bryophytes & 0.03 & 0.010 \\
\hline
\end{tabular}

\subsection{Analysis of the group structure}

Ecosim analysis gave similar results using different evaluation models (Tab. 3). In the checkerboard species and the $\mathrm{C}$-score, the observed index is not significantly higher than that obtained for the randomized matrices, suggesting that the community is not structured by competition (see par. 2.3.2). The V-ratio index was not significantly lower than that obtained in the null matrices. If competition was a significant force structuring the functional groups then the ratio of variance in species-richness among groups would be smaller relative to the null model. Finally, when competition was evaluated by counting the number of unique species-combinations within taxonomic groups (Combo index), the result was not significantly lower than that expected by chance.

Tab. 3. Ecosim analysis of the taxonomic group structure in springs of the Italian Alps. Co-occurrence indices were: Checker $=$ number of checkerboard species pairs; $\mathrm{C}$-score $=$ Stone and Robert's (1990) C-score; V-ratio = Schluter's (1984) variance ratio; $\mathrm{Combo}=$ number of unique species combinations. $p=$ significance of the difference between the co-occurrence indices in the observed (Obs) and simulated (Sim) matrices according with the hypothesis (see par 2.3.2).

\begin{tabular}{lccc}
\hline & Obs & Sim & $p$ \\
\hline C-Score & 3.08 & 3.26 & 0.849 \\
V-Ratio & 3.54 & 1.90 & 1.000 \\
Checker & 2172 & 2309 & 1.000 \\
Combo & 20.50 & 21.10 & 0.159 \\
\hline
\end{tabular}

\subsection{Congruency in species assemblage patterns across taxonomic groups}

Species-richness across photoautotrophic groups is not, or only weakly, correlated (Tab. 4), indicating that it is not possible to select only one group to predict species-richness of the others. The congruency analysis performed between the assemblages of the taxonomic groups indicated weak congruency (Tab. 5). However, stronger congruency was observed between lichens, bryophytes and diatoms, than between these groups and the algae (Fig. 2).

Tab. 4. Pearson correlation of species-richness between photoautotrophic groups in springs of the Italian Alps. Values within brackets give the significance level.

\begin{tabular}{lccc}
\hline$r$ Pearson & Algae & Diatoms & Lichens \\
\hline Diatoms & $-0.406(0.061)$ & & \\
Lichens & $-0.082(0.717)$ & $0.201(0.371)$ & \\
Bryophytes & $-0.028(0.903)$ & $0.345(0.116)$ & $0.406(0.061)$ \\
\hline
\end{tabular}

Tab. 5. Cross-taxa congruency in springs of the Italian Alps using community similarity determined by Mantel tests and performed with Primer software. The strength of is determined by the Rho-value, where 0 denoting no relationship, 1 denoting a very strong relationship. In brackets are the $p$-values.

\begin{tabular}{lccc}
\hline Taxon & Algae & Diatoms & Lichens \\
\hline Diatoms & $0.154(0.064)$ & & \\
Lichens & $0.165(0.024)$ & $0.329(0.001)$ & \\
Bryophytes & $0.220(0.015)$ & $0.463(0.001)$ & $0.231(0.006)$ \\
\hline
\end{tabular}

\section{DISCUSSION}

This study investigated the main photoautotrophic groups in Alpine springs and provided new insights into the diversity patterns of these organisms, which can be used for planning future strategies for the conservation of spring biodiversity. 


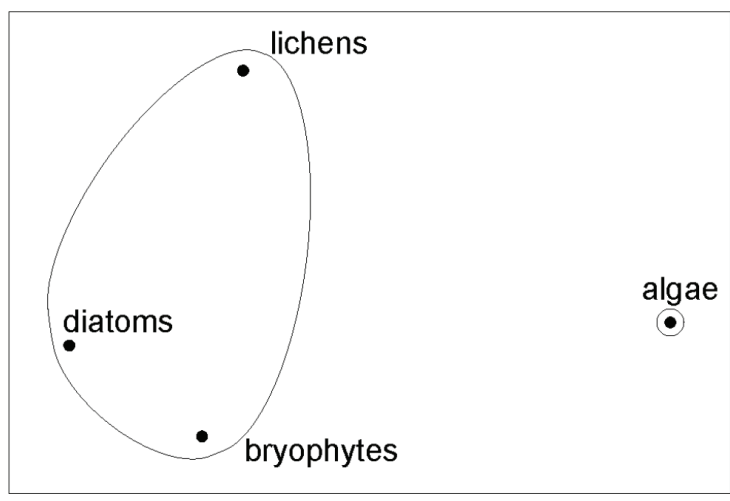

Fig. 2. Comparison of the clustering of taxa in springs of the Italian Alps using the Bray-Curtis similarity matrices. Secondstage multi-dimensional scaling (MDS) plot showing the relatedness of each taxonomic group. The grouped taxa were positively correlated at a fusion level of 0.2 . MDS stress-value $=0.01$.

With respect to substratum type, the four photoautotrophic groups showed similar patterns of diversity. Siliceous springs are more species-rich for diatoms, lichens and bryophytes, while only algae showed higher richness in carbonate springs. However, for all groups, carbonate and siliceous springs host different assemblages, indicating that both types of substrata contribute to the regional diversity of crenic photoautotrophs. Higher diversity in siliceous springs could be related to higher substratum stability and roughness that facilitate the establishment of epilithic organisms on siliceous rocks (Nascimbene \& Nimis 2006). For algae including cyanoprokaryotes, the lower hardness of the carbonate substratum might favour the establishment of several partially-endolithic species, e.g. crenic cyanobacteria (e.g., Leptolyngbya perforans). Spitale et al. (2009) suggested another explanation for the higher bryophyte richness in siliceous springs. They observed that, if the species requirement corresponds to the conditions under which they evolved, one might expect a larger speciespool of hygro-hydrophilous species on siliceous substrata, because siliceous substrata are generally damper (Michalet et al. 2002). The higher average moisture of siliceous substrata (than carbonate) might have promoted species differentiation among the hygro-hydrophilous species, in turn increasing the species-pool available to the crenic habitat.

Despite the fact that algae, diatoms, and bryophytes represent $90 \%$ of the photoautotrophic crenic biota, the percent contribution of freshwater lichens in individual springs (relative to their regional pool) is similar to that of diatoms and mosses, and higher than that of algae. However, in individual springs photoautotrophs form relatively species-poor communities, with a corresponding turnover of species among springs. This has important implications for conservation, suggesting that the protection of single sites is not an effective measure, and that a biodiversity conservation plan for crenic habitats must be developed at the regional level and include a network of sites on different substrata.

Interestingly, the co-occurrence indices revealed that, in individual springs, the local species composition (species co-occurrences compared between taxonomic groups) does not significantly differ from the null model. This suggests that neutral, stochastic processes are likely to be the most important mechanisms in the establishment of local assemblages. This is not to say that we exclude the importance of biotic interactions between taxonomic groups, but rather, that these forces are unlikely consistently to structure the crenic community. It is probable that the wide range of environmental gradients considered (mainly altitude and substratum reactivity) was the primary filter responsible for assembling the local community from the regional species pool. Once the subset species has been filtered by the environment, lichens, diatoms, bryophytes and algae might still interact, but probably only in particular situations. From our analysis, species interactions among taxonomic groups are unlikely to be the main force structuring the crenic assemblages.

Previous studies demonstrated generally poor congruence between different taxonomic groups in springs. For example, Virtanen et al. (2009), who compared bryophyte and insect assemblages in boreal springs, suggested that, although the overall compositional patterns of different taxonomic groups were significantly similar, the relative importance of environmental factors explaining their community composition differed strongly. The results implied that crenic bryophytes and insects were relatively poor surrogates for each other. Similar results were also obtained by Paavola et al. (2003) who compared fish, benthic macroinvertebrates and bryophytes in headwater streams. Our results agree with these studies, even though one might expect stronger surrogacy between more similar taxonomic groups: lichens, bryophytes, diatoms and algae are all autotrophic, while the other studies evaluated surrogacy between more diverse taxonomic groups. Despite the fact that significant relationships were found across almost all our taxonomic groups, suggesting some form of congruency (Tab. 5), we agree with Lovell et al. (2007) who used a minimum Rho-value of 0.75 as an additional constraint to the statistical significance. In this way, a taxonomic group might be considered a surrogate of another group only when the former represent at least the $75 \%$ of the latter. Photoautotrophs, despite sharing many habitat resources, are highly diverse in terms of life history and biological traits. Accordingly, this analysis revealed that any particular group surrogate would not adequately represent other photoautotrophic groups. In order to conserve photoautotrophs in springs it would therefore not be appropriate to use one group as a surrogate for overall photoautotrophic diversity. On the contrary, the weakness of cross-taxon congruency strongly supports the use of different taxonomic groups. 
In addition, given the different taxonomic composition in siliceous and carbonate Alpine springs, effective conservation management should include springs on different lithological substrata.

\section{ACKNOWLEDGEMENTS}

This work was supported by the CRENODAT Project (Biodiversity assessment and integrity evaluation of springs of Trentino - Italian Alps - and long-term ecological research, 2004-2008) financed by the University and Scientific Research Department of the Autonomous Province of Trento, Italy. We are grateful to the Editor, Ingrid Jüttner, to Péter Ódor, and to another anonymous Referee for their positive comments and for providing useful suggestions which improved the manuscript.

\section{REFERENCES}

Aboal, M., M. Puig \& M. Prefasi. 1998. Diatom assemblages in springs in Castellón province (Eastern Spain). Algological Studies, 90: 79-95.

Allan, J.D. 1975. Components of diversity. Oecologia, 18: 359-367.

Barquin J. \& M. Scarsbrook. 2008. Management and conservation strategies for coldwater springs. Aquatic Conserv. Mar. Freshw. Ecosyst., 18: 580-591.

Botosaneanu, L. 1998. Sources: aux portes du Styx. In: Botosaneanu, L. (Ed.), Studies in Crenobiology. The biology of springs and springbrooks. Backhuys, Leiden: 229-250.

Cantonati, M. 1998. Diatom communities of springs in the Southern Alps. Diatom Res., 13(2): 201-220.

Cantonati, M. 2008. Cyanoprokaryotes and algae other than diatoms in springs and streams of the Dolomiti Bellunesi National Park (Northern Italy). Algological Studies, 126: 113-136.

Cantonati, M. \& D. Spitale. 2009. The role of environmental factors, habitat, and microhabitat in shaping diatom assemblages in springs and streams of the Dolomiti Bellunesi National Park (south-eastern Alps). Fund. Appl. Limnol., 174: 117-133.

Cantonati, M., R. Gerecke \& E. Bertuzzi. 2006. Springs of the Alps, sensitive ecosystems to environmental change: From biodiversity assessments to long-term studies. Hydrobiologia, 562: 59-96.

Cantonati, M., E. Rott, P. Pfister \& E. Bertuzzi. 2007. Benthic algae in springs of the Alps: biodiversity and sampling methods. In: Cantonati, M., E. Bertuzzi \& D. Spitale (Eds), The spring habitat: biota and sampling methods. Monografie del Museo Tridentino di Scienze Naturali, 4: 77-112.

Cazaubon, A. \& T. Rolland. 1995. Essai de typologie des peuplements diatomiques des sources. Vie et milieu, 45: 315-319.

Clarke, K.R. \& R.N.Gorley. 2001. PRIMER v6: User manual/tutorial, PRIMER-E, Plymouth.

Cortini-Pedrotti, C. 2001. Flora dei muschi d'Italia. Sphagnopsida, Andreaeopsida, Bryopsida (I parte). Antonio Delfino Editore, Roma: 818 pp.

Cortini-Pedrotti, C. 2006. Flora dei muschi d'Italia. Bryopsida (II parte). Antonio Delfino Editore, Roma: 416 pp.

Di Sabatino, A., B. Cicolani \& R. Gerecke. 2003. Biodiversity and distribution of water mites (Acari, Hydrachnidia) in spring habitats. Freshwat. Biol. 48: 2163-2173.

Gotelli, N.J. 2000. Null model analysis of species co-occurrence patterns. Ecology, 81: 2606-2621.

Gotelli, N.J. \& G.L. Entsminger. 2001. EcoSim: Null models software for ecology. Version 7.0. Acquired Intelligence
Inc. \& Kesey-Bear. http://homepages.together.net/ gentsmin /ecosim.htm.

Hinterlang, D. \& D. Lischewski. 1996. Proceedings $1^{\text {st }}$ Symposium on Spring ecology and Conservation. Crunoecia, 5: $140 \mathrm{pp}$.

Howein, H. \& H. Schroeder. 2006. Geomorphologische Untersuchungen. In: Gerecke, R. \& H. Franz (Eds), Quellen im Nationalpark Berchtesgaden. Faunistisch-okologische Untersuchungen und Perspektiven für die langfristige Umweltbeobachtung. Nationalpark Berchtesgaden, Forschungsbericht, 51: 71-86.

Koleff, P., K.J. Gaston \& J.J. Lennon. 2003. Measuring beta diversity for presence-absence data. J. Anim. Ecol., 72: 367-382.

Lande, R. 1996. Statistics and partitioning of species diversity, and similarity among multiple communities. Oikos, 76: 5-13.

Legendre, P. \& L. Legendre. 1998. Numerical ecology. Second English edition. Elsevier Science B.V., Amsterdam: $853 \mathrm{pp}$.

Lovell, S., M. Hamer, R. Slotow \& D. Herbert. 2007. Assessment of congruency across invertebrate taxa and taxonomic levels to identify potential surrogates. Biol. Conserv., 139: 113-125.

Magurran, A.E. 2004. Measuring biological diversity. Blackwell Science Ltd: 256 pp.

McCune, B. \& M.J. Mefford. 1999. Multivariate analysis of ecological data. Version 4.25. MjM Software, Gleneden Beach, Oregon, US.

McCune, B. \& J.B. Grace. 2002. Analysis of ecological communities. MjM Software, Gleneden Beach, Oregon, US.

Michalet, R., Gandoy, C., Joud, D. \& J.P. Peges. 2002. Plant community composition and biomass on calcareous and siliceous substrates in the northern French Alps: comparative effects of soil chemistry and water status. Arct. Antarct. Alp. Res., 34: 102-113.

Nascimbene, J. \& P.L. Nimis. 2006. Freshwater lichens of the Italian Alps: a review. Ann. Limnol.-Int. J. Lim., 42: 27-32.

Nascimbene, J., Thüs, H., Marini. L. \& PL. Nimis. 2007. Freshwater lichens in springs of the eastern Italian Alps: floristics, ecology and potential for bioindication. Ann. Limnol.-Int. J. Lim., 43: 285-292.

Nimis, P.L. \& S. Martellos. 2008. ITALIC - The Information System on Italian Lichens. Version 4.0. University of Trieste, Dept. of Biology, IN4.0/1 (http://dbiodbs.univ. trieste.it/), December 2008.

Paavola, R.M.T., R. Virtanen, J. Heino \& P. Krevi. 2003. Are biological classifications of headwater concordant across multiple taxonomic groups? Freshwat. Biol., 8: 1912-1923.

Paton, J.A. 1999. The Liverwort Flora of the British Isles. Harley Books, Martins, Colchester, Essex: 626 pp.

Sabater, S. \& J.R. Roca. 1990. Some factors affecting distribution of diatom assemblages in Pyrenean springs. Freshwat. Biol., 24: 493-507.

Spitale, D. 2009. Spatial distribution of bryophytes along a moisture gradient: an approach using photosynthetic pigments as indicators of stress. Ecol. Res., 24(6): 1279-1286.

Spitale, D., Petraglia, A. \& M. Tomaselli. 2009. Structural equation model detects unexpected differences between bryophyte and vascular plant richness along multiple environmental gradients. J. Biogeog., 36: 745-755.

Stream Bryophyte Group. 1999. Roles of bryophytes in stream ecosystems. J. N. Am. Benthol. Soc., 18: 151-184.

Thűs, H. 2002. Taxonomie, Verbreitung ond Ökologie silicicoler subwasserflechten im auseralpinen Mitteleuropa. Bibliotheca Lichenologica, 83: 214 pp.

Virtanen, R., J. Ilmonen, L. Paasivirta \& T. Muotka. 2009. Community concordance between bryophyte and insect assemblages in boreal springs: a broad-scale study in isolated habitats. Freshwat. Biol., 54: 1651-1662. 